\title{
A spontaneous binge-like eating model in mice using unpredictable once weekly access to palatable diets
}

M. Murphy' ${ }^{1}$, D.J. Dijkstra ${ }^{1,2}$, J.S. Duncan ${ }^{1}$, G.W. Horgan ${ }^{3}$, J.G. Mercer ${ }^{1}$

\author{
${ }^{1}$ University of Aberdeen Rowett Institute, Foresterhill, Aberdeen AB25 2ZD, UK \\ ${ }^{2}$ current address: Department of Obstetrics and Gynaecology, University Medical Centre Groningen, \\ University of Groningen, Groningen, The Netherlands \\ ${ }^{3}$ Biomathematics \& Statistics Scotland, Rowett Institute, University of Aberdeen, Foresterhill
} Aberdeen AB25 2ZD, UK.

\section{$\underline{\text { Abstract }}$}

Many pre-clinical models of binge-like eating involve predictable, scheduled, access to a palatable diet high in fat (HF), where access may be preceded by anticipatory behaviour. Here, to introduce spontaneity into the binge-type consumption of palatable diets, mice were allowed two hours access on a random day once per week and at a random time within an eight hour window either side of the transition from dark phase to light phase. Despite normal intake of a stock diet prior to unpredictable access to HF diet, mice immediately initiated a substantial eating episode when presented with HF diet. Following this consumption, compensatory hypophagia was observed relative to stock diet-fed controls, and cumulative energy intakes converged. There were no effects of HF diet on body weight or body composition over a 12-week period. Binge-like consumption was also observed on unpredictable access to the complete liquid diet, chocolate Ensure, but not with a $10 \%$ sucrose solution. Binge-like responses to unpredictable access to HF diet or Ensure were similar in male and female mice, although there were effects of sex on caloric consumption from stock diet in the compensatory period following palatable diet intake, with higher intakes in females. The timing of the $2 \mathrm{~h}$ access period relative to light phase transition affected intake of palatable diets, but 
less robustly than the equivalent effect on stock diet intake during the same timed periods - the diurnal patterning of energy intake was diet sensitive. The large spontaneous binge-like consumption on unpredictable access to either solid or liquid palatable diets in mice of either sex offers the potential to combine these attributes with other manipulations where a developing obesity is part of the binge-like eating phenotype.

\section{Introduction}

Induction of binge-like consumption in rodent models requires that palatable food is available intermittently rather than continuously (Corwin \& Buda-Levin, 2004; Corwin, Avena \& Boggiano, 2011). One of the lines of development of pre-clinical binge-like eating models over the last $10-20$ years has settled on a protocol where palatable diet is available for $2 \mathrm{~h}$, either every day or on 3 days of each week; outwith this access period, animals are free to feed ad libitum on a bland diet (Barrett, Mercer \& Morgan, 2016). The variants on this 'limited access' model, which does not require prior food restriction (Corwin, 2004), have been designed to address some of the key diagnostic criteria for binge-eating disorder (BED; Mathes, Brownley, Mo \& Bulik, 2009;) set out in successive versions of the Diagnostic and Statistical Manual of Mental Disorders (http://www.dsm5.org/), published by the American Psychiatric Association. Binge-like episodes are discrete (within a 2 hour period; Wolfe, Baker, Smith \&Kelly-Weeder, 2009) and recurrent (at least weekly over 3 months). Rodent models can, by design, mimic both these episodic and recurrent features, as well as spontaneously exhibiting rapid consumption of an abnormally large amount of food when not hungry.

The early development of the limited access model consisted of timed access to vegetable fat (Corwin et al., 1998; Dimitriou, Rice \& Corwin, 2000), where, in rats, elevated energy intake from fat was compensated for by reduced intake of bland diet, giving rise to no change in cumulative energy intake or body weight. More recently, a $45 \% \mathrm{HF}$ pellet diet was employed in the same schedule, and 
binge-like eating resulted in weight gain in rats given daily access to HF diet compared with bland diet fed controls, despite self-restriction of bland diet intake in the intervening periods (Berner, Avena \& Hoebel, 2008). Our earlier studies with this model extended it to encompass both $45 \%$ and $60 \% \mathrm{HF}$ pellet diet, and a caloric liquid diet/supplement (Ensure ${ }^{\mathrm{TM}}$ or sucrose solution), each given daily at the same time each day to both mice and rats (Bake, Duncan, Morgan \& Mercer, 2013). With this manipulation, both rats and mice consumed more than $50 \%$ of their daily energy intake from HF diet during the $2 \mathrm{~h}$ access period, and with daily access there was generally an increase in body adiposity (Bake et al., 2013, Bake, Morgan \& Mercer, 2014; Bake et al., 2017).

The regimes employed in our earlier studies, in which periods of access to palatable diet were scheduled for the same time every day and stock diet was freely available for the remainder of the light:dark cycle, led to the development of food anticipatory activity (FAA; Patton \& Mistlberger, 2013) in our mouse model (Bake, Murphy, Morgan \& Mercer, 2014). Although relatively simple to deliver logistically in the animal facility, predictable scheduled access to palatable diet may not be the best mimic of aspects of human binge eating. Indeed, our studies also indicated that binge-like consumption of a palatable diet might be equally substantial on unpredictable access; specifically, mice that had scheduled access to HF diet withdrawn and then reinstated after a 7-day break, during which time overall intake and the diurnal patterning of stock pellet consumption returned to normal, responded immediately to the reintroduction of HF diet (Bake, Murphy, Morgan \& Mercer, 2014). This suggests that anticipation is not important in determining the size of the binge-like eating episode in this model, and, furthermore, that stomach capacity is not a limiting factor.

Consequently, in the current studies, we sought to incorporate spontaneity into the binge-like eating model by only providing unpredictable access to palatable food. A scenario of $2 \mathrm{~h}$ access to palatable diet once weekly over a 3-month period was selected to provide resonance with BED criteria, minimising the likelihood of animals being able to anticipate access, whilst providing access at least 
once per week. This unpredictable, randomised access should allow for expression of more spontaneous and impulsive behaviours.

We report three studies in mice applying unpredictable, once weekly, time-limited access to palatable diets. We conducted an initial characterisation of the model using HF pellet diet, before comparing responses of male and female mice to unpredictable access to HF diet, palatable liquid diet (Ensure) or sucrose solution, and the influence of timing within the light:dark cycle on energy intake both during and subsequent to a $2 \mathrm{~h}$ access period. Finally, the status of the neuroendocrine and reward-based signalling systems involved in energy balance were assessed coincident with, and following, binge-like feeding on HF diet.

\section{Methods}

\section{Animals and diet manipulations:}

Mice (C57BL/6, 7 weeks of age upon arrival from Charles River, Margate, UK) were habituated to the animal facility on a reversed $12 \mathrm{~h}$ light:12h dark cycle, and fed on a stock diet (CRM (P), 801722; Special Diets Services, Witham, UK; $22 \%$ protein, $69 \%$ carbohydrate, $9 \%$ fat by energy; $2.67 \mathrm{kcal} / \mathrm{g}$ ) for 2 weeks prior to the start of each study. Accordingly, mice were 9 weeks of age at the start of each experiment. A dim red light was on continuously in the animal room. All animals were individually housed after 1 week of acclimation in groups. Procedures were licensed under the Animals (Scientific Procedures) Act of 1986 and all studies were conducted with approval from the Rowett Institute Ethical Review Committee.

Three experiments were conducted during which mice were given unpredictable access to high fat (HF) diet (D12492; Research Diets, New Brunswick, NJ, USA; 60\% fat by energy, 20\% protein, 20\% carbohydrate; $5.24 \mathrm{kcal} / \mathrm{g}$;), or a palatable liquid diet (see Experiment 2), ad libitum for 2 hours only, 
once every 7 days, in a randomised schedule. Stock diet was freely available for the remaining 22 hours and throughout the 7-day period. As the day of access to palatable diet was randomly selected for all three Experiments, intervals could be greater or less than 7 days. On access days, the access period was also randomised within an $8 \mathrm{~h}$ window (4 hours either side of the dark/light transition). Accordingly, with lights going 'on' and 'off' at Zeitgeber time (ZT) 0 and 12, respectively, an access period beginning at, for example, ZT20 would mean that access would be from ZT20-ZT22, or the period $20-22 \mathrm{~h}$ after lights on (8-10h after lights off). The $8 \mathrm{~h}$ window during which palatable diet might be available thus spanned from ZT20 to ZT4, with start of access timed for ZT20-ZT2.

Body weights were measured twice a week, and daily food intake was calculated by manual weigh back on normal days and across the $2 \mathrm{~h}$ access period when animals were given access to a palatable diet in their home cage. When mice were given access to palatable diet during a $72 \mathrm{~h}$ feeding monitor session, the system (TSE PhenoMaster/LabMaster feeding/drinking monitor; TSE Systems, Bad Homburg, Germany) was programmed to measure food consumption every minute. Body composition (fat and lean tissue mass) was measured by EchoMRI ${ }^{\mathrm{TM}}$ (Echo Medical Systems, Houston, USA), immediately post mortem.

Experiment 1: 24 male C57BL/6 mice were assigned to 2 weight-matched groups of 12 animals. A control group (Stock) was fed stock pellet diet ad libitum, whereas the second group (HF diet) also had unpredictable access to HF pellets. Access to HF diet was provided on one randomised day per week, regardless of housing conditions (home cage or TSE cage). In each of the 12 weeks of the experiment, mice spent 3 consecutive days in the feeding/drinking monitor; this coincided with the access period on three occasions (weeks 4,8 and 12 ). Weekly access was provided on days $6,10,15$, $24,34,42,44,53,61,70,72$ and 81, and for 2 hours (Zeitgeber time at start of access period; ZT21 [dark], ZT0.5 [light], ZT22.5 [dark/light], ZT20.5 [dark], ZT1.5 [light], ZT0.75 [light], ZT1.25 [light], ZT23.25 [dark/light], ZT21 [dark], ZT20.5 [dark], ZT23.5 [dark/light] and ZT1.25 [light], respectively), 
during which time stock diet was withdrawn. Accordingly, TSE data were collected on day 24 starting at ZT20.5 (week 4), on day 53 at ZT23.25 (week 8), and on day 81 at ZT1.25 (week 12).

Experiment 2: 48 male and 48 female C57BL/6 mice were assigned to weight-matched groups of 12 animals and were given unpredictable ad libitum access once a week (days $6,14,19,23,31,36,45$, 51, 63, 67, 71 and 79) for 2 hours (ZT20.5 [dark], ZT23.5 [dark/light], ZT22.25 [dark/light], ZT2 [light], ZT23 [dark/light], ZT20.75 [dark], ZT0 [light], ZT23.25 [dark/light], ZT21.25 [dark], ZT0.75 [light], ZT1.5 [light] and ZT20 [dark]) to one of the following palatable diets: (i) $10 \%$ (w/v) sucrose solution (0.4kcal $/ \mathrm{ml}$ ), (ii) chocolate Ensure ${ }^{\mathrm{TM}}$ (a complete liquid nutritional supplement; Abbott Laboratories; $1.1 \mathrm{kcal} / \mathrm{ml}: 14 \%$ protein, $22 \%$ fat, $64 \%$ carbohydrate), or (iii) $60 \% \mathrm{HF}$ pellet. The study lasted for 12 weeks, during which time mice spent 3 consecutive days in the feeding/drinking monitor on three occasions (weeks 4, 8 and 12), with the access period occurring on the second day of monitoring.

Experiment 3: 64 male C57BL/6 mice were assigned initially to 2 weight-matched groups (Stock or HF diet; $n=32$ ). Weekly access to HF diet was randomised to days $6,9,19,23,35$ and 37 , and access periods were timed at ZT23.5 [dark/light], ZT20 [dark], ZT22.25 [dark/light], ZT2 [light], ZT23 [dark/light] and ZT20 [dark], respectively. Mice were housed in the feeding/drinking monitor on days 8-10 and 36-38. Eight mice from each of the Stock and HF groups (again approximately matched by weight) were killed by intraperitoneal injection of barbiturate anaesthetic at each of four different time points relative to the day 37 access period. The four time points were: 1 hour prior to access (ZT19), immediately at the end of $2 \mathrm{~h}$ access (ZT22), and 8 hours (ZT6) or 21 hours after end of the access period (next day, ZT19). Blood was collected by cardiac puncture into heparinised tubes and plasma was stored at $-80^{\circ} \mathrm{C}$ until analysis. Brains were frozen on dry ice and stored at $-80^{\circ} \mathrm{C}$ until cryostat sectioning. Plasma concentrations of leptin and insulin were assayed using commercially available enzyme-linked immunosorbent assay (ELISA) kits (Millipore, Billerica, MA, USA). The sensitivity of the leptin kit (cat. no. EZML-82K) was $0.05 \mathrm{ng} / \mathrm{ml}$, with an intra-assay variability of 
$10.8 \%$ and an inter-assay variability of $9.6 \%$, and that of the insulin kit (cat. no. EZRMI-13K) 0.1 $\mathrm{ng} / \mathrm{ml}$, with an intra-assay variability of $21.0 \%$ and an inter-assay variability of $7.8 \%$.

\section{In situ hybridisation:}

Coronal $15 \mu \mathrm{m}$ cryostat sections through the hypothalamus and forebrain were thaw-mounted onto sets of 10 poly-L-Lysine coated slides, and stored at $-80^{\circ} \mathrm{C}$ until analysis of mRNA expression, which was quantified by in situ hybridisation using established methods (Simmons, Arriza \& Swanson, 1989; Mercer et al., 1995). Hypothalamic sections (approximately caudal $-2.80 \mathrm{~mm}$ to rostral -1.70 mm relative to Bregma; Franklin \& Paxinos, 1997) included the arcuate nucleus (ARC). Forebrain sections (approximately rostral $1.18 \mathrm{~mm}$ to caudal $0.26 \mathrm{~mm}$ relative to Bregma) included the nucleus accumbens (NAcc). Antisense riboprobes $\left({ }^{35} \mathrm{~S}\right.$-labelled) to cloned fragments of the following cDNAs were used to hybridise brain sections: neuropeptide $Y(N P Y)$, agouti related peptide (AgRP), cocaine and amphetamine-regulated transcript (CART), proopiomelanocortin (POMC), leptin receptor, long form (OB-Rb), suppressor of cytokine signalling-3 (SOCS3), enkephalin (ENK), dynorphin (DYN), dopamine-2 receptor (D2-R), and dopamine-3 receptor (D3-R). Sections were fixed in paraformaldehyde, acetylated and hybridised overnight. After hybridisation and RNase A incubation, slides were dehydrated and apposed to autoradiographic film (BioMax MR; Kodak, Rochester, NY, USA). Autoradiographic images were analysed and quantified using Image ProPlus 7.0 software (Media Cybernetics, Bethesda, MA, USA). Four to six comparable sections spanning the ARC or NAcc were quantified from each animal. The area analysed within the NAcc was the combined core and shell.

\section{Statistics:}

Statistical analysis was performed using Sigmaplot 13.0 software or Genstat 19. Data are presented as mean \pm SEM. $P<0.05$ was considered statistically significant. In Experiment 1 , differences in body weight, weight gain and body composition were analysed by $\mathrm{t}$ test or Mann-Whitney Rank Sum test. 
Energy intake in $2 \mathrm{~h}$ or $24 \mathrm{~h}$ bins before, during and after the HF diet access period was analysed by Two Way Repeated Measures ANOVA with diet and time bin as factors. In Experiment 2, differences in body weight gain and body composition were analysed by One Way ANOVA or One Way ANOVA on Ranks. Home cage energy intake during $2 \mathrm{~h}$ access and TSE cage energy intake in $2 \mathrm{~h}, 12 \mathrm{~h}$ or $24 \mathrm{~h}$ ( $22 \mathrm{~h}$ at week 12 ) bins were analysed by 3-way hierarchical analysis of variance (ANOVA) with terms for diet, sex, week and all interactions, and a blocking term (random effect) for animal. In Experiment 3, differences in body weight, weight gain and body composition were analysed by t test, whereas differences in blood hormones and brain gene expression across the final access period were analysed by Two Way ANOVA with diet and time as factors, and with pairwise group comparisons using the Holm-Sidak test to correct for multiple comparisons.

\section{$\underline{\text { Results }}$}

Experiment 1: During the 12-week time-course, mice in the HF group received 12 unpredictable periods of free access to HF diet, one per week, each of 2-hours' duration. There was no effect on body weight (Stock - starting weight $24.5 \pm 0.3 \mathrm{~g}$, final weight $28.4 \pm 0.7 \mathrm{~g}$, weight gain $3.9 \pm 0.6 \mathrm{~g}$; HF diet - starting weight $24.5 \pm 0.4 \mathrm{~g}$, final weight $28.2 \pm 0.4 \mathrm{~g}$, weight gain $3.7 \pm 0.3 \mathrm{~g}$; all between group comparisons, $\mathrm{P}>0.05$ ) or post-mortem body composition (fat mass - Stock $2.5 \pm 0.2 \mathrm{~g}$; HF diet $2.7 \pm 0.2 \mathrm{~g}$; lean mass - Stock 20.8 $\pm 0.3 \mathrm{~g}$; HF diet 20.7 $\pm 0.3 \mathrm{~g}$; both between group comparisons, $\mathrm{P}>0.05$ ).

The typical structure of energy intake before, during and after an unpredictable $2 \mathrm{~h}$ period of access to HF diet is shown in Figure 1A, using TSE system data collected in week 12 as an exemplar; in week 12 , the access period occurred in the early light phase (ZT1.25-3.25), when feeding activity would normally be very low. Two-way RM ANOVA on the $24 \mathrm{~h}$ periods either side of the access period, and the $2 \mathrm{~h}$ access period itself, revealed significant effects of time interval $(F(2,42)=125.95, P<0.001)$ and an interaction between diet and time interval $(F(2,42)=87.38, P<0.001)$. Energy intake from stock 
diet during the $24 \mathrm{~h}$ preceding the access period was very similar in Stock and HF groups ( $\mathrm{t}=0.67$, $P>0.05)$, indicating that feeding on stock diet was unaffected by prior dietary experience. There was a rapid escalation of energy intake by the HF diet mice at the start of the access period. Intake was sustained at a high rate over the next 15-30 min, before declining in the second half of the access period (Fig. 1A). As expected, intake of stock diet by Stock mice was minimal during the access period - averaging $0.2 \mathrm{kcal}$ over the $2 \mathrm{~h}$ period (vs $7.7 \mathrm{kcal}$ of HF diet consumed by the HF group; Stock vs HF diet, $t=10.13, \mathrm{P}<0.001$; Fig. 1B). Energy intake from HF diet during the access period was approximately $75 \%$ of that consumed from stock diet during the previous $24 \mathrm{~h}$. In the $24 \mathrm{~h}$ following the access period, HF mice exhibited hypophagia on stock diet, relative to both the Stock group during the same time interval and their own intake during the $24 \mathrm{~h}$ prior to the access period $(\mathrm{t}=6.10$, $t=5.22$, both $P<0.001 ;$ Fig. $1 B$ ), such that the cumulative energy intake of the two groups converged over time (Fig. 1A). Taking into account both the access period and the post-access compensatory phase, there was no overall effect of HF diet on 50h energy intake encompassing the $24 \mathrm{~h}$ periods either side of the access period (Stock $23.0 \pm 1.3 \mathrm{kcal}$ vs HF diet $25.7 \pm 0.9 \mathrm{kcal} ; 2$-way RM ANOVA, $F(1,42)=2.99, P=0.10)$.

Experiment 2: Mice of both sexes were given $2 \mathrm{~h}$ unpredictable access to either a sucrose solution, Ensure liquid diet or pelleted HF diet once a week for 12 weeks. For males, body weights were closely matched, and weight gain across the study and post-mortem body composition was similar for all 4 diet groups (Suppl. Table S1). For females, starting body weights were again closely matched (Table S1), but there was an effect of diet on weight gain (one-way ANOVA, $F(3,44)=7.86, P<0.001$; Stock, 4.0 $\pm 0.2 \mathrm{~g}$; Sucrose, $4.3 \pm 0.2 \mathrm{~g}$; Ensure, $4.7 \pm 0.2 \mathrm{~g}$; HF diet, $3.4 \pm 0.1 \mathrm{~g}$ ) with significantly less weight gain in the HF group than in Ensure or Sucrose groups $(t=4.69,3.34 ; \mathrm{P}<0.001$ and $\mathrm{P}<0.01$, respectively). Despite these differences in weight gain, post-mortem body composition revealed no differences in body fat or lean mass due to diet (Suppl. Table 1), although the difference in lean mass approached significance $(F(3,44)=2.52, P=0.07)$, with $\mathrm{HF}$ female mice having the lowest lean mass. 


\section{Home cage energy intake during the access period}

Energy intake during the unpredictable weekly access periods was measured during 3 periods of housing in TSE cages (weeks 4, 8 and 12) and otherwise in the home cage (weeks 1, 2, 3, 5, 6, 7, 9, 10 and 11). Home cage energy intakes during $2 \mathrm{~h}$ access periods were analysed across 9 study weeks for 4 diets and in both sexes by 3-way analysis of variance (Fig. 2). Three access periods occurred in the late dark phase, three straddled the dark/light transition and three occurred in the early light phase (Fig. 2, key at top of figure). There was a significant effect of diet on caloric intake ( $F[3,87]=588.4$, $P<0.001)$, but no overall effect of sex, and no interaction between diet and sex - the effect of diet did not depend on sex. There was a significant effect of week on home cage energy intake $(F[8,695]=27.4, P<0.001)$. Further, a significant interaction between diet and week $(F[24,695]=6.9$, $P<0.001)$ indicated that the effect of week was different between diets. This finding reflects the reduced variability between weeks on the palatable diets. The interaction between sex and week approached significance $(F[8,695]=1.86, P=0.06)$, but there was no diet.week.sex interaction. In summary, in the Stock diet groups, both males and females exhibited the highest intakes during dark phase access (means: male 1.8kcal; female $1.9 \mathrm{kcal}$ ) and the lowest intakes during light phase access (means; male $0.6 \mathrm{kcal}$; female $0.6 \mathrm{kcal}$ ). Home cage energy intakes in the Sucrose group exceeded $0.9 \mathrm{kcal}$ at all time points (means: dark phase, male $1.6 \mathrm{kcal}$, female $1.3 \mathrm{kcal}$; light phase, male $1.0 \mathrm{kcal}$, female $1.1 \mathrm{kcal})$. A substantial and consistent increase in access period energy intake was observed with Ensure with a minimum intake of $3.8 \mathrm{kcal}$ (means: dark phase, male $5.3 \mathrm{kcal}$, female $5.4 \mathrm{kcal}$; light phase, male $4.2 \mathrm{kcal}$, female $4.6 \mathrm{kcal})$. In the HF diet group, energy intake during the access period was further elevated, with a minimum intake of $5.1 \mathrm{kcal}$ (means: dark phase, male $7.1 \mathrm{kcal}$, female $6.9 \mathrm{kcal}$; light phase, male $7.1 \mathrm{kcal}$, female $5.9 \mathrm{kcal})$.

\section{TSE cage energy intake during the access period}


Minute-by-minute analysis of energy intake in TSE cages on week 4 (access period early in the light phase) is shown in Suppl. Figure 1. Cumulative energy intake for male mice during the access period averaged $6.0 \mathrm{kcal}, 2.7 \mathrm{kcal}, 0.4 \mathrm{kcal}$ and $0.1 \mathrm{kcal}$ for HF pellet, Ensure liquid, Sucrose solution and Stock diet, respectively (Suppl. Figure $1 \mathrm{~A}$ ). For HF diet, $74 \%$ of cumulative energy intake was consumed in the first hour of access; the equivalent data for Ensure, Sucrose and Stock diet were $68 \%, 84 \%$ and $52 \%$, respectively. Energy intake in female mice during the access period averaged $4.5 \mathrm{kcal}, 2.0 \mathrm{kcal}$, $0.5 \mathrm{kcal}$ and $0.4 \mathrm{kcal}$ for HF diet, Ensure, Sucrose and Stock diet, respectively (Suppl. Figure 1B), with $83 \%, 72 \%, 81 \%$ and $91 \%$ of this consumed in the first hour.

Cumulative energy intake in TSE cages was assessed in 3 time bins - $2 \mathrm{~h}$ access period, $12 \mathrm{~h}$ following the access period, and $24 \mathrm{~h}$ (or $22 \mathrm{~h}$ on week 12 ) following the access period - on 3 study weeks (week 4, light phase access; week 8, light/dark transition access; week 12, dark phase access) for all 4 diets and in both sexes (Fig. 3). Energy intake data was analysed by 3-way ANOVA.

For the $2 \mathrm{~h}$ access period in the TSE cages (Fig. 3 upper panel), there was an effect of diet on energy intake $(F[3,87]=403.1, P<0.001)$, but no effect of sex and no interaction between diet and sex. Energy intake from HF diet was always $>100 \%$ greater than from Ensure, independent of week or sex, whereas energy intake from Ensure was always greater than that from Sucrose or Stock. There was an effect of week $(F[2,168]=38.6, P<0.001)$, with dark phase intake (week 12) being highest and light phase intake (week 4) lowest. There was also an interaction between diet and week $(F[6,168]=6.4$, $\mathrm{P}<0.001$ ), indicating, as for the home cage access data, that effect of week was different between diets. Although there was no interaction between sex and week, there was an effect of sex on the diet.week interaction $(F[6,168]=2.9, P<0.01)$, suggesting that the differences between weeks on the palatable diets were stronger in females.

TSE cage energy intake during the $12 \mathrm{~h}$ period following access 
In the $12 \mathrm{~h}$ period following access (Fig. 3 middle panel), there was an effect of diet on energy intake $(F[3,87]=15.4, P<0.001)$, due to compensatory hypophagia, especially following HF diet access. The overall effect of sex approached significance $(F[1,87]=2.8, P=0.097)$, and there was an interaction between diet and sex $(F[3,87]=4.0, P<0.05)$, suggesting that differences between diets were stronger in female mice. There was an effect of week $(F[2,165]=21.9, P<0.001)$, with light phase intake (week 4) being highest and light/dark transition lowest (week 8), and there was an interaction between diet and week $(F[6,165]=2.4, P<0.05)$, suggesting that differences between diets were greater in weeks 4 and 8 than in week 12.

\section{TSE cage energy intake during the $24 / 22 \mathrm{~h}$ period following access}

In the $24 \mathrm{~h}$ ( $22 \mathrm{~h}$ for week 12 data) following the access period (Fig. 3 lower panel), compensatory hypophagia was still apparent and there was an effect of diet on energy intake ( $F[3,87]=22.8$, $P<0.001)$, an effect of $\operatorname{sex}(F[1,87]=11.4, P=0.001)$, and a significant interaction between diet and sex $(F[3,87]=3.5, P<0.05)$. Energy intakes were highest in females and differences between diets were stronger in female mice. There was an effect of week $(F[2,164]=29.9, P<0.001)$, with light phase intake being highest and dark phase lowest, as well as interactions between diet and week $(F[6,164]=2.2, P<0.05)$, and sex and week $(F[2,164]=9.0, P<0.001)$.

Experiment 3: Mice in the HF diet group had 6 unpredictable periods of access to HF diet, each of 2hours' duration across the 38-day experiment, and were killed at 4 prescribed times (ZT19, ZT22, ZT6 and next day, ZT19) relative to the final access period late in the dark phase (ZT20). Minute-byminute recordings of energy intake in TSE cages for each timed group are shown in Figure 4. Body weights did not differ between Stock and HF diet groups (Stock - starting weight $24.7 \pm 0.2 \mathrm{~g}$, final weight (day 36) $27.9 \pm 0.3 \mathrm{~g}$, weight gain $3.1 \pm 0.2 \mathrm{~g}$; HF diet - starting weight $24.7 \pm 0.2 \mathrm{~g}$, final weight $28.1 \pm 0.3$, weight gain $3.4 \pm 0.2 \mathrm{~g}$; all between group comparisons, $\mathrm{P}>0.05)$, and there were no differences in post-mortem body composition, despite a trend for HF diet mice to have higher body 
fat mass (fat mass - Stock 2.6 $\pm 0.1 \mathrm{~g}$, HF diet 2.9 $\pm 0.1 \mathrm{~g}, \mathrm{t}=1.72, \mathrm{P}=0.09$; lean mass - Stock $19.2 \pm 0.3 \mathrm{~g}, \mathrm{HF}$ diet $19.1 \pm 0.3 g, t=0.21, P>0.05)$.

Analysis of leptin and insulin in terminal blood plasma samples revealed no effect of diet or timing, and no interactions between these factors (2-way ANOVA; all P>0.05; Table 1). Gene expression analysis in the ARC and NAcc indicated very limited effects of dietary manipulation and timing relative to the access period. Representative autoradiographic images depicting hypothalamic gene expression are shown in Figure 5. There was no statistically significant effect of diet on any of the genes, although there was a trend for NPY gene expression to be lower in HF groups compared to those fed stock diet throughout $(F(1,47)=3.48, P=0.068)$. This was accompanied by a trend towards a negative correlation between terminal plasma leptin and NPY gene expression in the ARC across the final access period $(r=0.248 ; P=0.071)$. There was an effect of time relative to the access period on expression of three of the genes examined (Table $1 ; \mathrm{P}<0.05)$. Expression of leptin receptor (OB$\mathrm{Rb})$ mRNA in the ARC was higher at next day, ZT19 than at ZT6 (8h after access). In the NAcc, expression levels of ENK and D2R were higher at ZT22 than at next day, ZT19 and ZT19, respectively. There was also a trend for DYN expression to be influenced by time $(F(3,50)=2.46, P=0.073)$, with a similar pattern to that observed for ENK (Table 1).

\section{Discussion}

We developed and characterised the unpredictable access/binge-like eating model having observed anticipatory activity with regular (daily) scheduled periods of access to palatable diet (Bake, Murphy, Morgan \& Mercer, 2014). These previous reports of binge-like consumption of palatable diets on a scheduled (daily) regime are thus in line with the definition of Berner et al. (2008) of "consuming more of a given food in a discrete period of time than would normally be consumed under similar circumstances". With unpredictable access, spontaneous binge-like consumption occurs without the 
opportunity for prior adjustment in either energy intake or behaviour. Unsurprisingly, given the compensatory hypophagia observed following once weekly binge-like feeding, unpredictable access did not give rise to weight gain or increased adiposity. As with a number of similar dietary manipulations, even where energy dense palatable diets are provided daily or several times a week (e.g. Corwin et al., 1998), overt obesity is not part of the resulting phenotype. Male and female mice reacted similarly to unpredictable access to palatable diet in terms of caloric consumption, although sex-related differences emerged in terms of energy intake during the post-access period. Of the diets tested, the HF pellet diet and, to a lesser extent, the liquid diet Ensure increased energy intake during the unpredictable access period, whereas for Sucrose solution intakes did not consistently exceed energy intake from stock pellet diet.

The modified dietary manipulation used in the current studies, allowing mice to 'react' to the sudden appearance of the palatable diet despite feeding normally on stock diet up until that point, gave rise to similar levels of binge-like consumption, at least for HF diet and Ensure, as a completely predictable daily access model. Using such a scheduled access model in which palatable diets were available for $2 \mathrm{~h}$ in the middle of the dark phase (Berner et al., 2008), we have previously compared HF diet, Ensure or $12.5 \%$ Sucrose solution and observed that mice can obtain $86 \%, 45 \%$ and $24 \%$, respectively, of their normal daily energy intake from these sources during a $2 \mathrm{~h}$ access period (Bake et al., 2013). Although similar levels of energy intake were achieved from unpredictable access to HF diet and Ensure in the current studies, with characteristics similar to binge-like eating, intake of Sucrose solution was not consistently higher than energy intake from Stock pellet, and did not reach the level of caloric intake compared to control that has been suggested as a working definition of binge-like consumption (2-fold vs control, Perello, Valdivia, Romero \& Raingo, 2014; >25\% of daily calories in $1 \mathrm{~h}$, Halpern et al., 2013). This outcome may reflect the comparatively low energy density of the sucrose solution, although an effect mediated via stomach volume appears unlikely given the volume of Ensure consumed during the access period. It may be that provision of a complete or 
near-complete diet is require for manifestation of excessive caloric intake under the precise conditions employed in the current study.

Two-hour periods of unpredictable access to palatable diet were timed to four hours either side of the dark/light transition. It is presumed that, depending on the precise timing, mice were unlikely to have been hungry and might have been approaching satiety when palatable diet was presented, having been feeding normally on stock diet during the dark phase. This speculation is supported by the very similar intakes of stock diet observed in Stock and HF mice in the run up to the access period in Experiment 1 (Fig. 1A,B), and the minimal caloric intake of Stock mice during the access period in Experiment 3 (Fig. 4). This presumed physiological state satisfies one of the criteria for BED diagnosis in humans, namely that subjects are not hungry at the start of the binge period. There was a strong diurnal patterning of home cage energy intake during different $2 \mathrm{~h}$ access periods in the Stock diet group (Fig. 2, top panels; high intake in the dark phase, low in the light phase). The significant interaction between diet and week identified by 3-way ANOVA of data in Figure 2 indicated that the effect of week on caloric intake during the access period was different between diets. Accordingly, the variability between weeks, i.e. due to position in the light/dark cycle, although still apparent with the palatable diets, was reduced. This suggests that the reward value of the palatable diets partially overrides normal diurnal feeding patterns. The probable involvement of the reward system makes it likely that despite the unpredictable nature of the weekly access to palatable diet, prior/accumulating experience and learning could influence feeding behaviours across the studies. In addition to effects on feeding during the access period, which were also observed in TSE data (Fig. 3 upper panel), the timing of access relative to the transition between dark and light phases affected subsequent 24/22h energy intake in Experiment 2, even taking into account the underestimate of energy intake on week 12 due to the final $22 \mathrm{~h}$ bin. Week effects on $24 / 22 \mathrm{~h}$ post-access intake were the opposite of those observed for $2 \mathrm{~h}$ access period intake. Accordingly, energy intake from HF diet during the $2 \mathrm{~h}$ access period was approximately $80 \%, 122 \%$ 
and $139 \%$ of energy intake from stock diet in the subsequent 24 h period on weeks 4,8 and 12 , respectively, for males, and approximately $65 \%, 101 \%$ and $145 \%$, respectively, for females. The equivalent data for Ensure were $24 \%, 32 \%$ and $54 \%$ for males, and $20 \%, 36 \%$ and $54 \%$ for females (Fig 3).

The outcomes of the HF diet manipulation employed in our studies were broadly similar to those reported with once weekly access to a $40 \% \mathrm{HF}$ diet, but where the access period was $24 \mathrm{~h}$ (Czyzyk, Sahr \& Statnick, 2010). Male C57BL/6 mice were offered a free choice of HF diet and chow every seventh day, with only chow available for the intervening 6 days. The $24 \mathrm{~h}$ access regime induced binge-like eating behaviour, although energy intake in the first 2.5h of access (mid-light cycle; Czyzyk et al., 2010) was only equivalent to one-third of the total daily caloric intake of control mice that were not on the $24 \mathrm{~h}$ access protocol, indicating that take-up was slower than in the more constrained protocol in the current studies. Across the whole $24 \mathrm{~h}$ access period, mice consumed approximately twice the calories (all from HF diet) of controls fed the choice diet throughout the study. However, there was compensation for this elevated intake with mice reducing their consumption of chow the following day; overall weekly energy intake was less than that of HF diet controls, and body weight, body composition and serum leptin did not differ from controls fed chow throughout (Czyzyk et al., 2010).

An increasing number of studies have investigated sex-specificity on the outcomes of dietary and energy balance manipulations in preclinical models, for example, in weight gain, loss, and subsequent regain upon switching between $\mathrm{HF}$ diet (DIO), caloric restriction on low fat diet, and refeeding ad libitum on HF diet (Benz et al., 2012). The evidence for comparable responses between male and female mice to unpredictable access to palatable diets (Expt. 2) is important to the translational potential of the model. Despite the similarities in intake during the access period, there were other sex-related outcomes in Experiment 2, including stronger differences between diets and 
between weeks in female mice, and higher energy intakes from stock diet in females in the postaccess period. While eating disorders are more prevalent in women than men, BED is the most common eating disorder in males, with a gender ratio less biased towards females (Davies, 2015). Further investigation of the common relative to sex-specific features of binge-like eating models is required since previous studies have observed higher rates of occurrence of a binge-like eating phenotype in female rats (Klump, Racine, Hildebrandt, Sisk, 2013), and binge-like behaviour in female but not male mice (Carlin et al., 2016). Sex differences have also been reported in other eating disorder models, energetic manipulations, and in FAA. Accordingly, male mice have been reported to have a greater susceptibility to activity-based anorexia than females (Achamrah et al., 2017), with different patterns of physical activity when access to food is restricted. Sexually divergent responses were also reported in rats subjected to chronic stress, food restriction and intermittent access (2h) to chocolate Ensure (Lenglos, Mitra, Guevremont \& Timofeeva, 2013); restraint stress decreased body weight gain in male rats, but not in females, an effect that was blunted by intermittent access to Ensure. FAA, induced by restricted feeding, and which we have previously shown is also expressed prior to scheduled access to HF diet with otherwise ad libitum access to stock diet (Bake, Murphy, Morgan \& Mercer, 2014), is also more pronounced in male mice than in females (Li et al., 2015; Michalik, Steele \& Mistlberger, 2015).

Binge-like eating responses during unpredictable access to palatable diets were similar in male and female mice in the current study with the exception of a sex.diet.week interaction indicating that differences between weeks on the palatable diets in TSE cages appeared stronger in females. During the post-access compensatory period, a similar effect was observed, with differences between diets or between weeks appearing stronger in females, as well as intakes being higher overall in females in the $24 / 22 \mathrm{~h}$ bin (Fig. 3). Compensation for hyperphagia on palatable diets has been reported frequently, but only occasionally in the context of possible sex-related differences. For example, in our earlier studies with scheduled daily access, male rats and mice consumed $63 \%$ and $86 \%$ of their 
average daily intake from HF diet, and $49 \%$ and $45 \%$ from Ensure during $2 \mathrm{~h}$ (Bake et al., 2013). Such substantial caloric intakes markedly reduced energy intake from stock diet during the remainder of the $24 \mathrm{~h}$ period, although this compensation was incomplete and total caloric intake was generally still elevated in the schedule-fed groups (Bake et al., 2013; Bake, Murphy, Morgan \& Mercer, 2014; Bake, Morgan \& Mercer, 2014; Bake et al., 2017). Similar outcomes were observed in separate but closely related studies when HF diet was provided daily or on 3 days per week (Berner et al., 2008). Substantial compensation was also observed when male mice were allowed weekly $24 \mathrm{~h}$ access to a HF diet (Czyzyk et al., 2010), and when male and female rats were given scheduled daily access to sweetened condensed milk (SCM) (Hume, Jachs \& Menzies, 2016), although compensation was less efficient when larger amounts of SCM were offered to, and consumed by, male rats (Hume et al., 2016). This ability to compensate at lower SCM intake levels was not affected by irregular presentation times within the daily light/dark cycle (Hume et al., 2016).

Our previous studies with the same diets (HF diet, Ensure, Sucrose; Bake et al., 2013), provided on a predictable daily schedule, demonstrated that there were no changes in expression of energy balance genes in the hypothalamic ARC or reward-related genes in the forebrain NAcc in the run up to the access period that could be interpreted as driving the consumption of large binge-like meals. Furthermore, differences in hypothalamic gene expression that were observed during the scheduled access period and coincident with binge-like eating on HF diet in mice (decreased NPY, increased CART during the $2 \mathrm{~h}$ access period; Bake et al., 2017) appeared to reflect body adiposity and blood leptin levels rather than appetite drive. In the current studies, we sampled tissues at 4 time points relative to the final access period. The lack of a clear effect of diet on energy balance genes with unpredictable access was consistent with the absence of any anticipatory behavioural component or clear body phenotype. Nevertheless, the weak effect of diet on NPY gene expression (Table 1), supported by a weak negative relationship between NPY and plasma leptin in HF mice with marginally higher body fat mass, is consistent with presumed counter-regulatory responses 
observed previously with DIO on ad libitum access to HF diet (Mercer \& Archer, 2008), and with scheduled feeding on HF diet (Bake et al., 2013; Bake et al., 2017). The current findings for rewardrelated gene expression (DYN, ENK, D2R, D3R) in the NAcc, while possibly reflecting diurnal regulation of gene expression, are also consistent with outcomes prior to and during daily scheduled access to HF diet (Bake et al., 2013; Bake et al., 2017), with no effect of diet. Similar outcomes have been reported for male C57BL/6J mice in a $1 \mathrm{~h} \mathrm{HF}$ diet access model (Halpern et al., 2013), where there were no changes in D1R or D2R gene expression the NAcc shell compared to chow fed controls at the time when palatable diet would be expected on the daily schedule, although a small reduction in D2R gene expression was recently reported in binge-like eating prone rats $5 \mathrm{~h}$ prior to accustomed $1 \mathrm{~h}$ access to vegetable fat in a limited access paradigm (Chawla, Cordner, Boersma \& Moran, 2017). Furthermore, in rats, ENK and DYN gene expression in the NAcc shell/core were unaltered $24 \mathrm{~h}$ after a daily palatable diet access, although there was a reduction in DYN mRNA in the medial prefrontal cortex (Blasio, Steardo, Sabino \& Cottone, 2013). Despite these findings, a body of evidence links components of the endogenous reward system, and specifically the dopamine (e.g. Johnson \& Kenny, 2010; Valdivia et al., 2015) and opioid (e.g. Kelley et al., 2003; Cottone, Sabino, Steardo \& Zorrilla, 2008; Blasio et al., 2013) systems to binge-like eating on various palatable diets in different access models. However, the involvement of these systems in an unpredictable access scenario remains to be established.

The unpredictable access/spontaneous access model has the advantage of provoking binge-like consumption in both sexes on either HF pellet or liquid Ensure. The binge-like episode on palatable diets is substantial, with a major proportion of normal daily caloric intake consumed in $2 \mathrm{~h}$ despite $\mathrm{ad}$ libitum feeding on stock diet up to the point of access. The dietary manipulation could allow compensatory processes and homeostatic/hedonic interactions to be examined, in this case in the absence of the complication of feedback from increased body adiposity, and avoids anticipatory processes. The model meets many of the criteria used to assess applicability of pre-clinical models to 
the human condition (Corwin \& Buda-Levin, 2004; Czyzyk et al., 2010) - short-term but substantial over-consumption relative to controls, that is repeatable over extended periods of time, not dependent upon food deprivation or prevented by satiety, and with freely-expressed compensatory behaviour. Immediate intake on access (the binge-like episode) is greater than that in other studies where access could be considered unpredictable, i.e. 24h access every seventh day (Czyzyk et al., 2010). It is unsurprising that the current manipulation, which is accompanied by compensatory reductions in later intake on stock diet, does not induce significant weight gain or changes in body composition. This phenotype is shared with some of the earlier 'limited access' studies from which it has been developed (Corwin et al., 1998; Czyzyk et al., 2010; Corwin et al., 2011). Since elevated body weight is strongly correlated with BED (Davis, 2015), this could potentially represent a major limitation, although, as alluded to above, there may also be advantages for specific mechanistic studies. Nevertheless, the spontaneity of the feeding response observed here, providing an immediate consummatory reaction to the environment, is a characteristic that could potentially be incorporated into other pre-clinical binge-like eating models that do go on to develop a phenotype that includes obesity and metabolic comorbidities, such as those induced by stress-induced hyperphagia (Razzoli, Pearson, Crow \& Bartolomucci, 2017). Combining the positive attributes of different pre-clinical models could allow development of better mimics of the human condition for mechanistic investigation.

\section{Acknowledgments}

The authors are grateful for funding from the Scottish Government Strategic Research Programme, and from the European Union Seventh Framework Programme (FP7/2007-2013) under grant agreement 245009 (NeuroFAST). 


\section{$\underline{\text { References }}$}

1. Achamrah, N., Nobis, S., Goichon, A., Breton, J., Legrand, R., do Rego, J. L., de Rego, J. C., Dechelotte, P., Fetissov, S. O., Belmonte, L., \& Coeffier, M. (2017). Sex differences in response to activity-based anorexia model in C57BI/6 mice. Physiology \& Behavior, 170, 1-5.

2. Bake, T., Baron, J., Duncan, J. S., Morgan, D. G. A., \& Mercer, J. G. (2017). Arcuate nucleus homeostatic systems reflect blood leptin concentration but not feeding behavior during scheduled feedign on high-fat diet inmice. Journal of Neuroendocrinology, 25, 357-371.

3. Bake, T., Duncan, J. S., Morgan, D. G. A., \& Mercer, J. G. (2013). Arcuate nucleus homeostatic systems are not altered immediately prior to the scheduled consumption of large, bingetype meals of palatable solid or liquid diet in rats and mice. Journal of Neuroendocrinology, $25,357-371$.

4. Bake, T., Morgan, D. G. A., \& Mercer, J. G. (2014). Feeding and metabolic consequences of scheduled consumption of large, binge-type meals of high fat diet in the Sprague Dawley rat. Physiology and Behavior, 128, 70-79.

5. Bake, T., Murphy, M., Morgan, D. G. A., \& Mercer, J. G. (2014). Large, binge-type meals of high fat diet change feeding behaviour and entrain food anticipatory activity in mice. Appetite 77C, 60-71.

6. Barrett, P., Mercer, J. G., \& Morgan, P. J. (2016). Preclinical models for obesity research. Disease Models \& Mechanisms, 9, 1245-1255.

7. Benz, V., Bloch, M., Wardat, S., Bohm, C., Maurer, L., Mahmoodzadeh, S., Wiedmer, P., Spranger, J., Foryst-Ludwig, A., \& Kintscher, U. (2012). Sexual dimorphic regulation of body weight dynamics and adipose tissue lipolysis. PLoS One, 7, e37794.

8. Berner, L. A., Avena, N. M., \& Hoebel, B. G. (2008). Bingeing, self-restriction, and increased body weight in rats with limited access to a sweet-fat diet. Obesity, 16(9), 1998-2002.

9. Blasio, A., Steardo, L., Sabino, V., \& Cottone, P. (2013). Opioid system in the medial prefrontal cortex mediates binge-like eating. Addition Biology doi:10.1111/adb.12033. 
10. Carlin, J. L., McKee, S. E., Hill-Smith, T., Grissom, N. M., George, R., Lucki, I., \& Reyes, T. M. (2016). Removal of high fat diet after chronic exposure drives binge behavior and dopaminergic dysregulation in female mice. Neuroscience 326, 170-170.

11. Chawla, A., Cordner, Z.A., Boersma, G., \& Moran, T. H. (2017) Cognitive impairment and gene expression alterations in a rodent model of binge eating disorder. Physiology \& Behavior 180, 78-90.

12. Corwin, R. L. (2004). Binge-like eating induced by limited access in rats does not require energy restriction on the previous day. Appetite 42, 139-142.

13. Corwin, R. L., Avena, N. M., \& Boggiano, M. M. (2011). Feeding and reward: Perspectives from three rat models of binge eating. Physiology and Behavior, 104, 87-97.

14. Corwin, R. L., \& Buda-Levin, A. (2004). Behavioral models of binge-type eating. Physiology and Behavior, 82, 123-130.

15. Corwin, R. L., Wojnicki, F. H. E., Fisher, J. O., Dimitriou, S. G., Rice, H. B., \& Young, M. A. (1998). Limited access to a dietary fat option affects ingestive behavior but not body composition in male rats. Physiology and Behavior, 65(3), 545-553.

16. Cottone, P., Sabino, V., Steardo, L., Zorrilla, E. P. (2008). Opioid-dependent anticipatory negative contrast and binge-like eating in rats with limited access to highly preferred food. Neuropsychopharmacology, 33, 524-535.

17. Czyzyk, T. A., Sahr, A. E., \& Statnick, M. A. (2010). A model of binge-like eating behavior in mice that does not require food deprivation of stress. Obesity, 18, 1710-1717.

18. Davis, C. (2015). The epidemiology and genetics of binge eating disorder (BED). CNS Spectrums 20, 522-529.

19. Dimitriou, S. G., Rice, H. B., \& Corwin, R. L. (2000). Effects of limited access to a fat option on food intake and body composition in female rats. International Journal of Eating Disorders, 28(4), 436-445. 
20. Franklin, K. B. J., \& Paxinos, G. (1997). The Mouse Brain in Stereotaxic Coordinates. San Diego: Academic Press.

21. Halpern, C. H., Tekriwal, A., Santollo,J., Keating, J. G., Wolf, J. A., Daniels, D., \& Bale, T. L. (2013 ). Amelioration of binge eating by nucleus accumbens shell deep brain stimulation in mice involves D2 receptor modulation. The Journal of Neuroscience 33, 7122-7129.

22. Hume, C., Jachs, B., \& Menzies, J. (2016). Homeostatic responses to palatable food consumption in satiated rats. Obesity 24, 2126-2132.

23. Johnson, P. M., \& Kenny, P. J. (2010). Dopamine D2 receptors in addiction-like reward dysfunction and compulsive eating in obese rats. Nature Neuroscience 13, 635-641.

24. Kelley, A. E., Will, M. J., Steininger, T. L., Zhang, M., \& Haber, S. N. (2003). Restricted daily consumption of a highly palatable food (chocolate Ensure ${ }^{\circledR}$ ) alters striatal enkephalin gene expression. European Journal of Neuroscience, 18, 2592-2598.

25. Klump, K. L., Racine, S., Hildebrandt, B., \& Sisk, C. L. (2013). Sex differences in binge eating patterns in male and female adult rats. International Journal of Eating Disorders, 46, 729736.

26. Lenglos, C., Mitra, A., Guevremont, G., \& Timofeeva, E. (2013 ). Sex differences in the effects of chronic stress and food restriction on body weight gain and brain expression of CRF and relaxin-3 in rats. Genes, Brain and Behaviour, 12, 370-387.

27. Li, Z., Wang, Y., Sun, K. K., Wang, K., Sun, Z. S., Zhao, M., \& Wang, J. (2015). Sex-related difference in food-anticipatory activity of mice. Hormones and Behavior, 70, 38-46.

28. Mathes, W. F., Brownley, K.A., Mo, X., \& Bulik, C. M. (2009). The biology of binge eating. Appetite 52, 545-553.

29. Mercer, J. G., \& Archer, Z. A., (2008). Putting the diet back into diet-induced obesity: dietinduced hypothalamic gene expression. European Journal of Pharmacology 585, 31-37.

30. Mercer, J. G., Lawrence, C. B., Beck, B., Burlet, A., Atkinson, T., \& Barrett, P. (1995). Hypothalamic NPY and prepro-NPY mRNA in Djungarian hamsters: Effects of food 
deprivation and photoperiod. American Journal of Physiology - Regulatory Integrative and Comparative Physiology, 269, R1099-R1106.

31. Michalik, M., Steele, A. D., \& Mistlberger, R. E. (2015). A sex difference in circadian foodanticipatory rhythms in mice: interaction with dopamine D1 receptor knockout. Behavioral Neuroscience, 129, 351-360.

32. Patton, D. F., \& Mistlberger, R., (2013). Circadian adaptations to meal timing: neuroendocrine mechanisms. Frontiers in Neuroscience, 7, 185. doi: 10.3389/fnins.2013.00185.

33. Perello, M., Valdivia, S., Romero, G. G., \& Raingo, J. (2014). Considerations about rodent models of binge eating episodes. Frontiers in Psychology 5, doi:10.3389/fpsyg.2014.00372.

34. Razzoli, M., Pearson, C., Crow, S., \& Bartolomucci, A. (2017). Stress, overeating, and obesity: insights from human studies and preclinical models. Neuroscience and Biobehavioral Reviews, 76, 154-162.

35. Simmons, D. M., Arriza, J. L., \& Swanson, L. W. (1989). A complete protocol for in situ hybridization of messenger RNAs in brain and other tissues with radiolabeled singlestranded RNA probes. Journal of Histotechnology 12, 169-181.

36. Valdivia, S., Cornejo, M. P., Reynaldo, M., De Francesco, P. N., \& Perello, M. (2015). Escalation in high fat intake in a binge eating model differentially engages dopamine neurons of the ventral tegmental area and requires ghrelin signaling. Psychoneuroendocrinology, 60, 206-216.

37. Wolfe, B. E., Baker, C. W., Smith, A. T., \& Kelly-Weeder, S. (2009). Validity and utility of the current definition of binge eating. International Journal of Eating Disorders, 42, 674-686.

\section{Figure legends}


Figure 1: Energy intake (kcal) of male C57BL/6 mice from week 12 of Experiment 1. Mice were given unpredictable access to HF diet for $2 \mathrm{~h}$ once every 7 days (HF diet) or were held on a stock diet throughout (Stock). Data shown are from the twelfth and final period of HF diet access which occurred on day 81 , during the early light phase (ZT1.25-ZT3.25) when mice were housed in the TSE feeding monitor: (A) minute-by-minute cumulative energy intake over $71 \mathrm{~h}$ where access to HF diet is marked by the rectangle; (B) energy intake from stock diet in $24 \mathrm{~h}$ bins immediately before or after the $2 \mathrm{~h}$ access period and from stock diet or HF diet during the $2 \mathrm{~h}$ access period. Histograms depict substantially elevated energy intake during the $2 \mathrm{~h} \mathrm{HF}$ diet access period and subsequent compensatory hypophagia on stock diet $\left({ }^{* * *} \mathrm{P}<0.001\right.$ vs Stock at same time interval; \#\#\# $\mathrm{P}<0.001$ vs HF diet in 24h before access). Data are means (+standard error in B), $n=12$.

Figure 2: Home cage energy intake (kcal) of male (left hand column) or female (right hand column) C57BL/6 mice in Experiment 2 during nine unpredictable periods of $2 \mathrm{~h}$ access to the following palatable diets: $10 \%$ sucrose solution (row 2), Ensure liquid (row 3), HF pellet diet (row 4). Access periods occurred randomly once every 7 days, whereas the Stock groups (row 1 ) were fed stock pellet throughout. Symbols at the top of each panel depict the approximate timing of palatable diet access on the week specified ( $\bullet$ dark during the whole access period, o light during the whole access period, 1 transition between dark and light during the access period). Data are means+standard error, $\mathrm{n}=12$.

Figure 3: Energy intake (kcal), analysed as three timed bins (the $2 \mathrm{~h}$ access period and the $12 \mathrm{~h}$ and 24/22h bins immediately following), of male (left hand column) or female (right hand column) C57BL/6 mice from weeks 4, 8 and 12 of Experiment 2. Mice were given unpredictable access to one of the following palatable diets: $10 \%$ sucrose solution, Ensure liquid or HF pellet diet for $2 \mathrm{~h}$ once every 7 days, or were held on a pellet stock diet throughout. Data shown are from the fourth, eighth and twelfth periods of palatable diet access which occurred on days 23,51 and 79 , during the early 
light phase (ZT2-ZT4), the dark-light transition (ZT23.25-ZT1.25), and the late dark phase (ZT20ZT22), respectively, when mice were housed in the TSE feeding monitor. Symbols at the top of each panel depict the approximate timing of palatable diet access on the week specified (• dark during the whole access period, $\mathrm{O}$ light during the whole access period, $\mathbf{O}$ transition between dark and light during the access period). Data are means+standard error, $n=12$.

Figure 4: Minute-by-minute cumulative energy intake (kcal) of male C57BL/6 mice from week 6 of Experiment 3. Feeding data were collected during housing in TSE cages for the 2 days running up to $2 \mathrm{~h}$ unpredictable access to HF diet and terminating at prescribed times (ZT19, ZT22, ZT6 and $\mathrm{ZT} 19(+24 \mathrm{~h}))$ relative to the access period $(\mathrm{ZT20}-\mathrm{ZT} 22)$ in the late dark phase. Data are means, $\mathrm{n}=8$. The HF diet access period is indicated by the black shaded bar.

Figure 5: Representative autoradiographic images of hypothalamic expression of energy balance related genes in stock diet and HF diet-fed mice.

Supplementary Figure 1: Minute-by minute feeding data from the week 4 access period of Experiment 2 showing cumulative energy intake (kcal) of (A) male or (B) female C57BL/6 mice housed in TSE cages and given $2 \mathrm{~h}$ unpredictable access to $10 \%$ sucrose solution, Ensure liquid or HF pellet diet. On week 4, the palatable diet access period was early in the light phase. Data are means, $\mathrm{n}=12$. 
Table 1: Quantification of serum hormones (leptin, insulin) and mRNA gene expression in the arcuate nucleus (ARC) or nucleus accumbens (NACC) of C57BL/6 mice in Experiment 3. Mice were killed at 4 time points relative to unpredictable access to high fat (HF) diet for 2 hours (ZT20-ZT22). Brain sections were hybridized with riboprobes for NPY, AgRP, CART, POMC, leptin receptor (OB-Rb) or SOCS3 (all ARC), the endogenous opioids, ENK and DYN (NACC), or the dopamine receptors, D2-R and D3-R (NAcC). Expression was normalized to Control ZT19 $(=100)$ for each probe. Data are means $\pm \mathrm{SE}$, with statistical analysis by 2-way ANOVA.

\begin{tabular}{|c|c|c|c|c|c|c|c|c|}
\hline & \multicolumn{2}{|c|}{ ZT19 } & \multicolumn{2}{|c|}{ ZT22 } & \multicolumn{2}{|c|}{ ZT6 } & \multicolumn{2}{|c|}{ next day, ZT19 } \\
\hline & Stock & HF diet & Stock & HF diet & Stock & HF diet & Stock & HF diet \\
\hline $\begin{array}{l}\text { Leptin } \\
\text { (ng/ml) }\end{array}$ & $2.0 \pm 0.3$ & $1.9 \pm 0.5$ & $2.0 \pm 0.4$ & $1.9 \pm 0.4$ & $1.1 \pm 0.3$ & $2.4 \pm 0.5$ & $2.3 \pm 0.3$ & $2.5 \pm 0.4$ \\
\hline \multirow[t]{2}{*}{$\begin{array}{l}\text { Insulin } \\
\text { (ng/ml) }\end{array}$} & $0.5 \pm 0.1$ & $0.7 \pm 0.3$ & $0.7 \pm 0.1$ & $0.7 \pm 0.3$ & $0.7 \pm 0.2$ & $0.7 \pm 0.1$ & $0.7 \pm 0.2$ & $0.5 \pm 0.1$ \\
\hline & \multicolumn{2}{|c|}{ ZT19 } & \multicolumn{2}{|c|}{ ZT22 } & \multicolumn{2}{|c|}{ ZT6 } & \multicolumn{2}{|c|}{ next day, ZT19 } \\
\hline$\underline{A R C}$ & Stock & HF diet & Stock & HF diet & Stock & HF diet & Stock & HF diet \\
\hline NPYa & $100 \pm 5.3$ & $94.2 \pm 5.7$ & $94.5 \pm 9.2$ & $101.4 \pm 5.3$ & $110.1 \pm 12.5$ & $95.9 \pm 6.2$ & $124.5 \pm 10.8$ & $90.5 \pm 12.5$ \\
\hline AgRP & $100 \pm 6.0$ & $103.3 \pm 9.03$ & $108.3 \pm 6.9$ & $124.6 \pm 12.3$ & $98.0 \pm 13.4$ & $92.3 \pm 9.06$ & $113.7 \pm 14.8$ & $106.8 \pm 15.2$ \\
\hline CART & $100 \pm 11.0$ & $119.8 \pm 15.5$ & $124.6 \pm 14.1$ & $98.9 \pm 11.1$ & $108.1 \pm 10.1$ & $123.9 \pm 10.6$ & $97.1 \pm 10.4$ & $108.0 \pm 6.1$ \\
\hline POMC & $100 \pm 10.8$ & $116.5 \pm 10.9$ & $131.4 \pm 12.6$ & $115.9 \pm 20.0$ & $99.9 \pm 12.6$ & $130.3 \pm 14.0$ & $99.4 \pm 7.9$ & $88.6 \pm 8.0$ \\
\hline$O B-R b^{b}$ & $100 \pm 7.2$ & $101.7 \pm 12.0$ & $110.4 \pm 18.3$ & $96.4 \pm 11.1$ & $74.4 \pm 4.3$ & $70.6 \pm 8.8$ & $135.3 \pm 23.5$ & $99.7 \pm 6.4$ \\
\hline SOCS3 & $100 \pm 16.4$ & $141.8 \pm 15.7$ & $123.4 \pm 19.9$ & $103.1 \pm 28.1$ & $96.8 \pm 15.1$ & $145.6 \pm 23.5$ & $172.0 \pm 38.0$ & $157.6 \pm 20.9$ \\
\hline \multicolumn{9}{|l|}{ NAcc } \\
\hline$D N^{c}$ & $100 \pm 6.9$ & $109.6 \pm 9.0$ & $132.2 \pm 11.9$ & $117.1 \pm 8.3$ & $117.2 \pm 7.0$ & $116.6 \pm 4.6$ & $110.1 \pm 9.0$ & $106.0 \pm 6.1$ \\
\hline ENK $^{d}$ & $100 \pm 6.5$ & $106.1 \pm 6.5$ & $123.0 \pm 5.6$ & $109.8 \pm 8.1$ & $109.7 \pm 3.3$ & $96.7 \pm 10.5$ & $91.3 \pm 5.0$ & $100.1 \pm 4.0$ \\
\hline $\mathrm{D} 2 \mathrm{R}^{\mathrm{e}}$ & $100 \pm 2.2$ & $106.0 \pm 4.2$ & $126.5 \pm 6.4$ & $110.2 \pm 6.6$ & $104.5 \pm 5.8$ & $107.4 \pm 3.8$ & $106.7 \pm 6.6$ & $114.0 \pm 4.9$ \\
\hline D3R & $100 \pm 11.5$ & $102.6 \pm 20.3$ & $118.3 \pm 15.4$ & $118.8 \pm 13.5$ & $79.2 \pm 11.0$ & $95.6 \pm 6.0$ & $105.1 \pm 18.6$ & $98.9 \pm 9.1$ \\
\hline
\end{tabular}

a trend effect of diet; Stock $>H F$ diet; $F(1,47)=3.48, P=0.068$

${ }^{b}$ significant effect of time; $F(3,46)=4.66, P=0.006$; next day, ZT19>ZT6

' trend effect of time; $F(3,50)=2.46, P=0.073$

${ }^{d}$ significant effect of time; $F(3,47)=3.40, P=0.025 ; Z T 22>$ next day, ZT19

e significant effect of time; $F(3,48)=3.07, P=0.037 ; Z T 22>Z T 19$ 


\section{Supplementary data}

Table S1: Experiment 2 - initial and final body weight and post-mortem body composition (means $\pm S E$ )

\begin{tabular}{|l|c|c|c|c|c|c|c|c|}
\hline & \multicolumn{4}{|c|}{ Male } & \multicolumn{3}{c|}{ Female } \\
\hline & Stock & Sucrose & Ensure & HF & Stock & Sucrose & Ensure & HF \\
\hline $\begin{array}{l}\text { Initial body } \\
\text { weight (g) }\end{array}$ & $24.5 \pm 0.3$ & $24.5 \pm 0.2$ & $24.4 \pm 0.2$ & $24.3 \pm 0.3$ & $19.1 \pm 0.2$ & $19.1 \pm 0.3$ & $19.0 \pm 0.2$ & $19.1 \pm 0.2$ \\
\hline $\begin{array}{l}\text { Final body } \\
\text { weight (g) }\end{array}$ & $30.4 \pm 0.6$ & $29.7 \pm 0.6$ & $29.7 \pm 0.4$ & $30.6 \pm 1.2$ & $23.1 \pm 0.2$ & $23.4 \pm 0.2$ & $23.6 \pm 0.4$ & $22.5 \pm 0.2$ \\
\hline $\begin{array}{l}\text { Body weight } \\
\text { change (g) }\end{array}$ & $5.9 \pm 0.4$ & $5.2 \pm 0.6$ & $5.2 \pm 0.4$ & $6.3 \pm 1.2$ & $4.0 \pm 0.2$ & $4.3 \pm 0.2$ & $4.7 \pm 0.2$ & $3.4 \pm 0.1^{\text {a,b }}$ \\
\hline Fat mass (g) & $3.9 \pm 0.4$ & $3.3 \pm 0.3$ & $3.4 \pm 0.3$ & $3.5 \pm 0.6$ & $2.1 \pm 0.1$ & $2.0 \pm 0.1$ & $2.2 \pm 0.1$ & $2.0 \pm 0.1$ \\
\hline Lean mass (g) & $20.7 \pm 0.2$ & $20.3 \pm 0.1$ & $20.5 \pm 0.2$ & $20.5 \pm 0.3$ & $16.4 \pm 0.2$ & $16.6 \pm 0.2$ & $16.3 \pm 0.1$ & $16.0 \pm 0.2$ \\
\hline
\end{tabular}

a $P<0.001$ vs Ensure

b $P<0.01$ vs Sucrose 
FIGURE 1: MURPHY ET AL.,

A

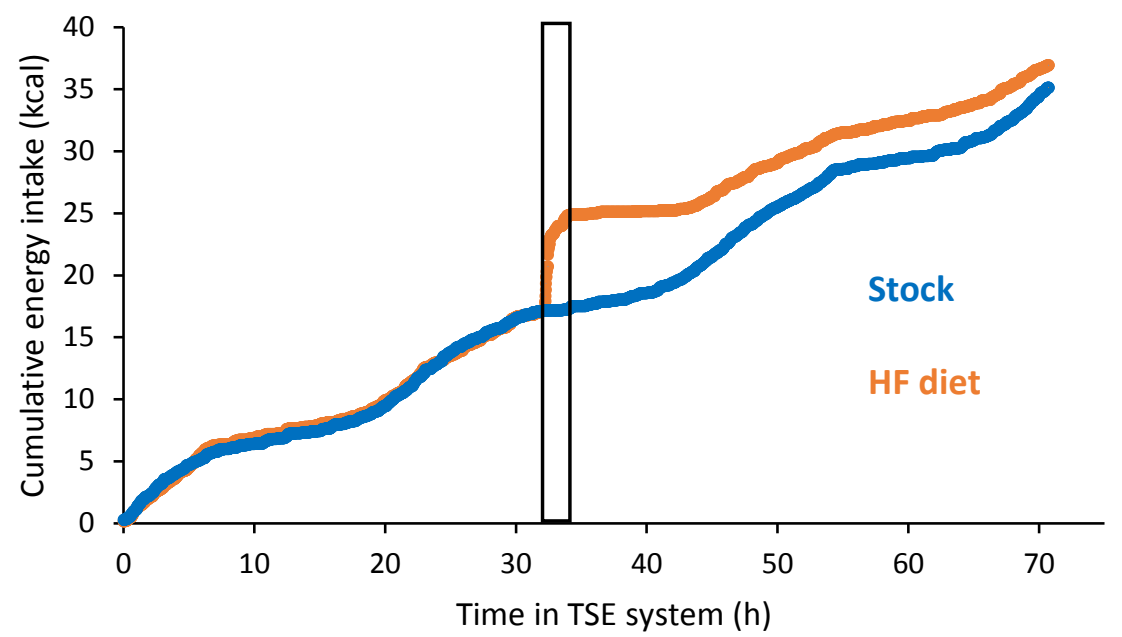

B

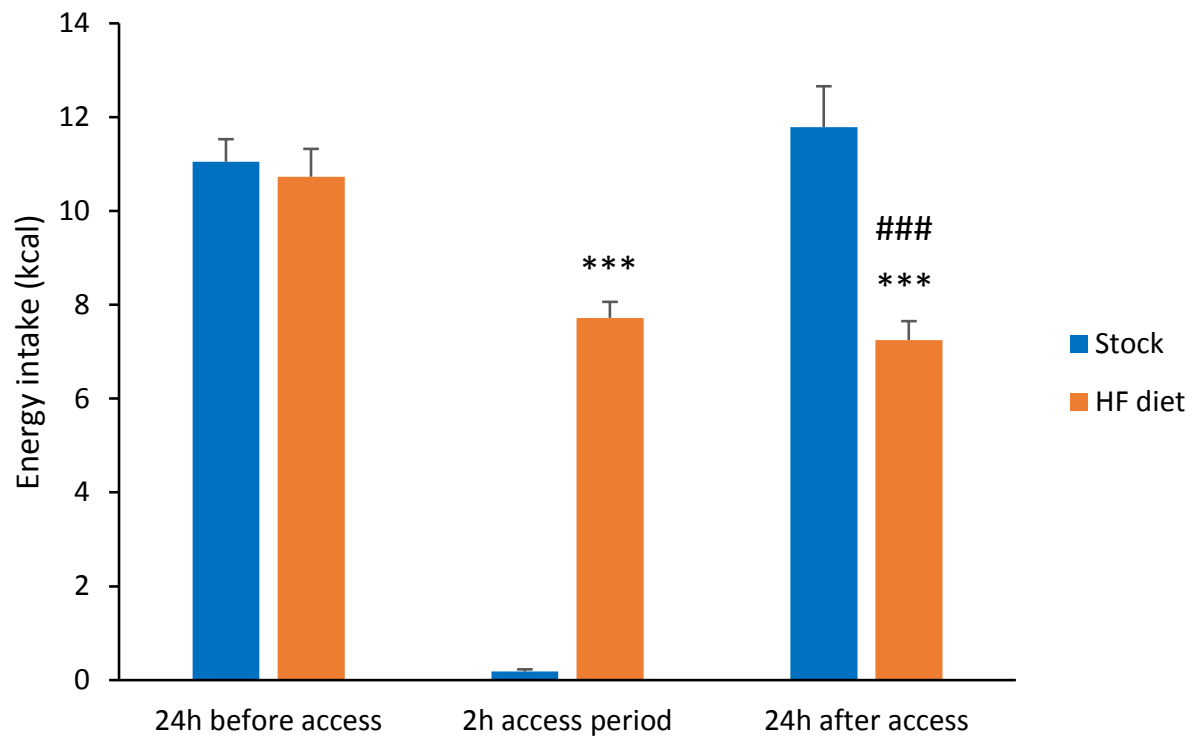


male $\bigcirc \oslash \oslash \oslash 00000$ female $00 \oslash \oslash 00000$
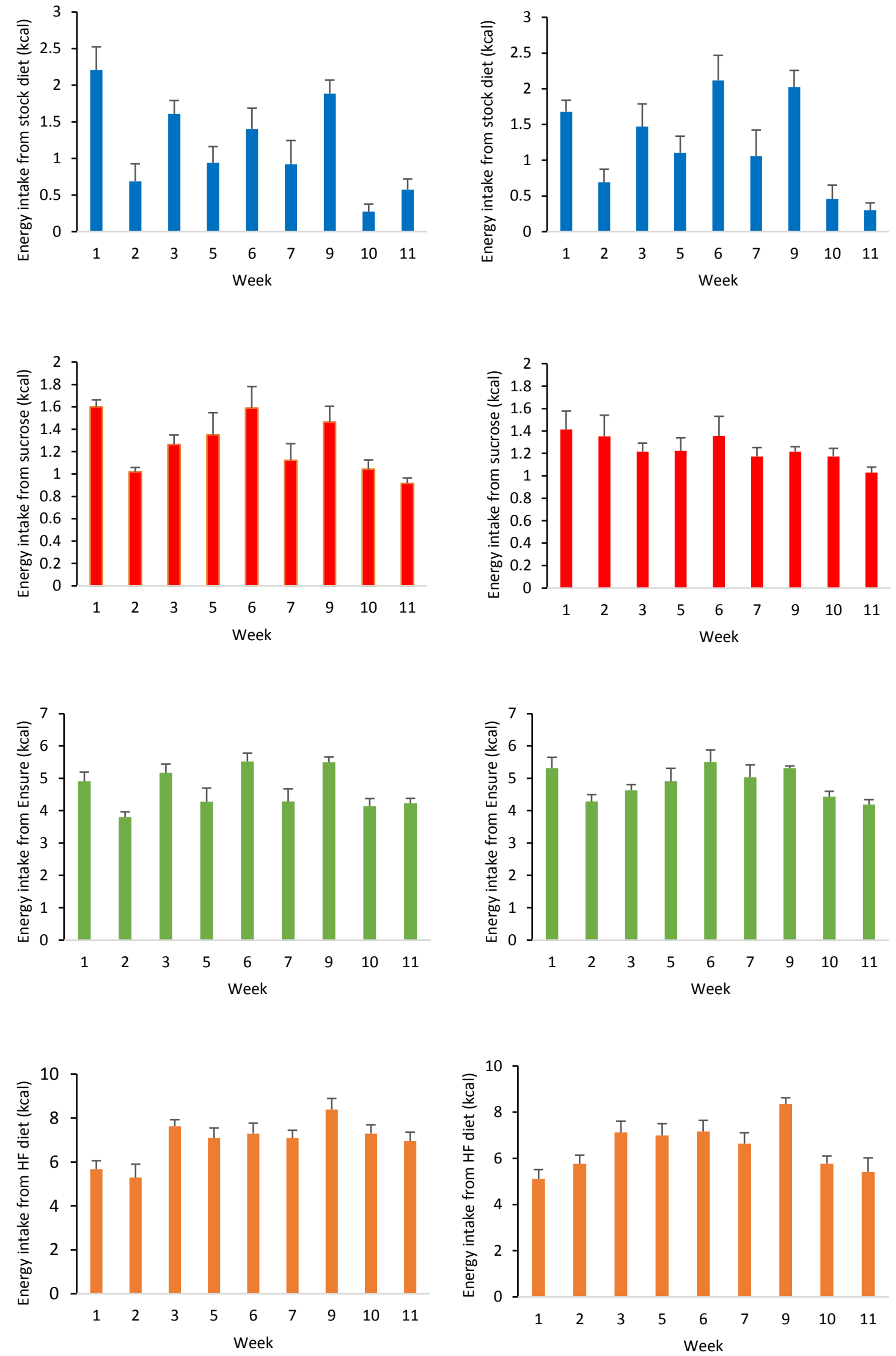

FIGURE 2: MURPHY ET AL., 
FIGURE 3: MURPHY ET AL.,

MALE

FEMALE
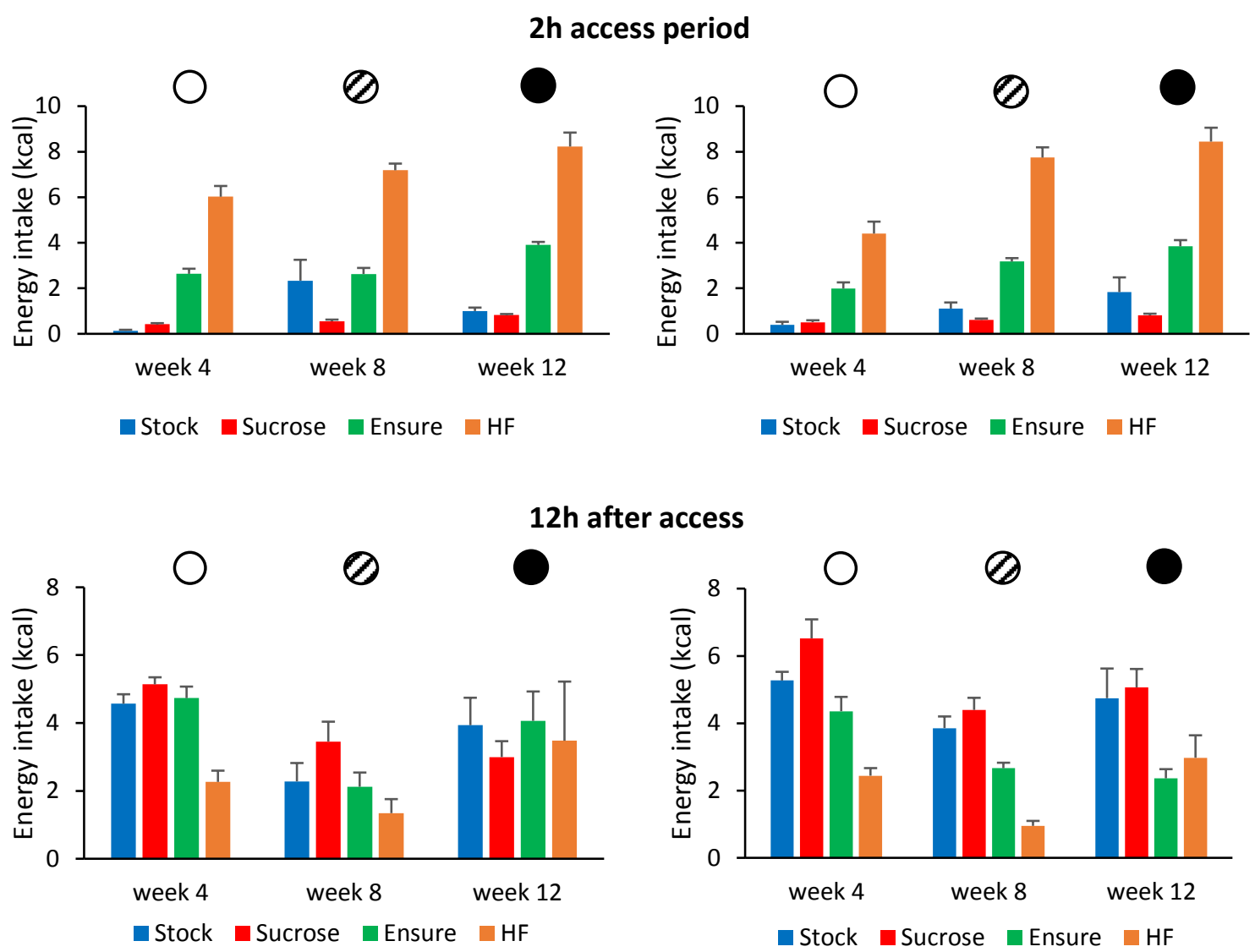

$\mathbf{2 4 / 2 2 h}$ after access
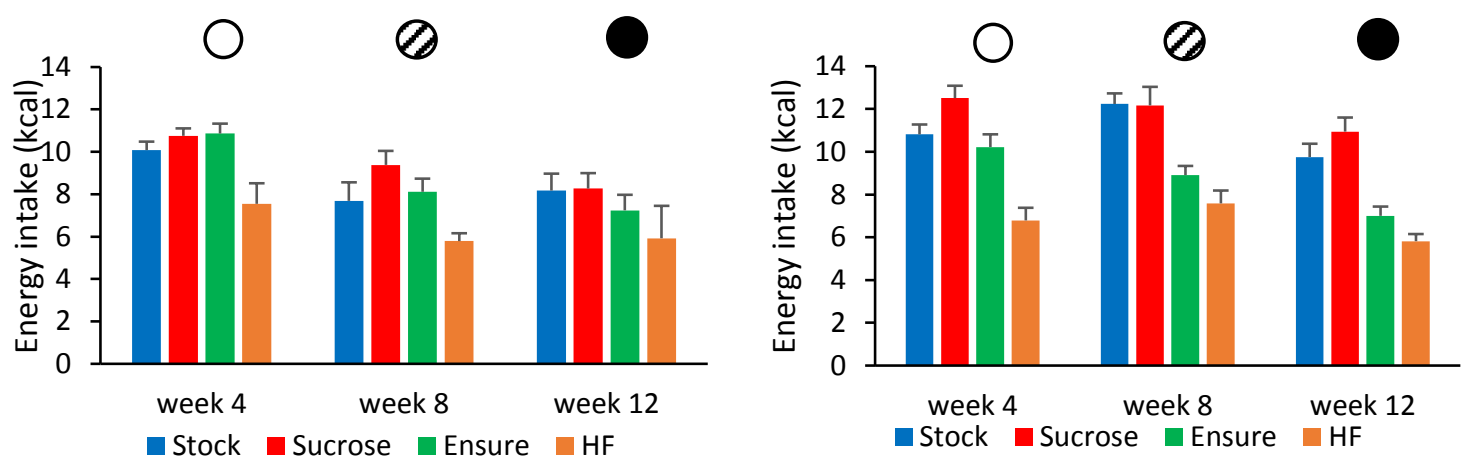
FIGURE 4: MURPHY ET AL.,

A

ZT19

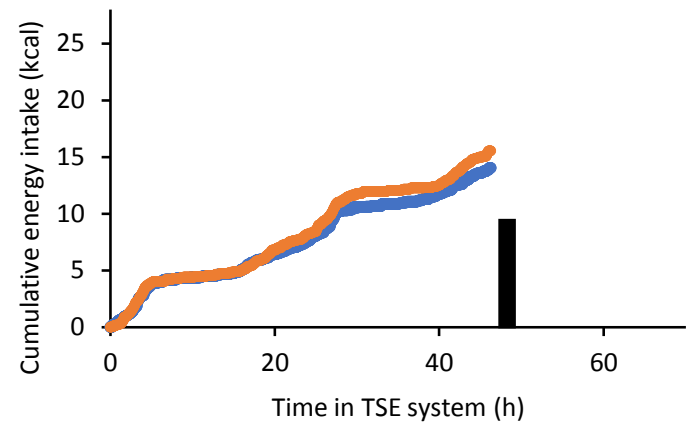

- Stock - HF diet

C $\quad$ ZT6

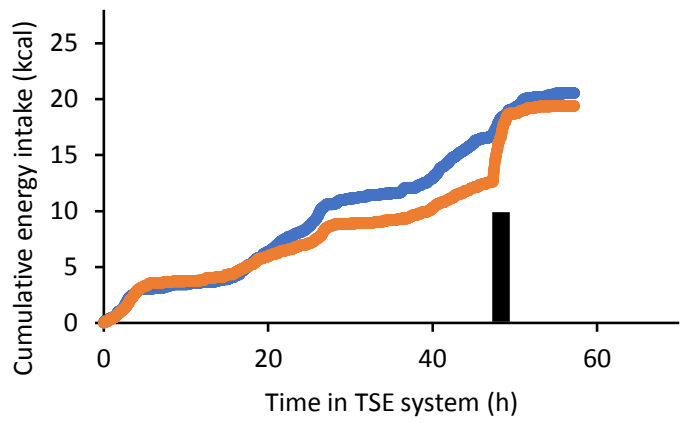

- Stock - HF diet
B $\quad$ ZT22

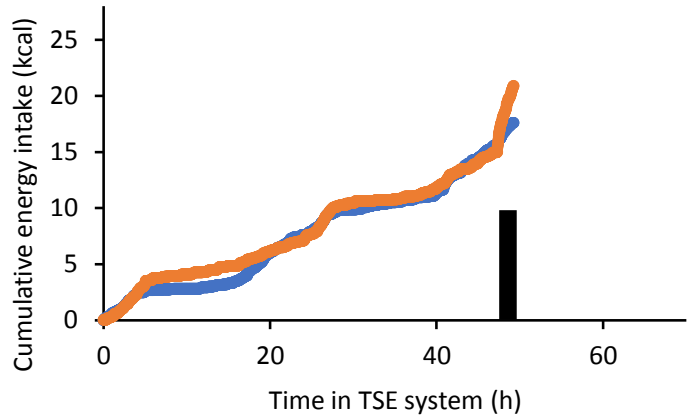

- Stock - HF diet

D next day ZT19

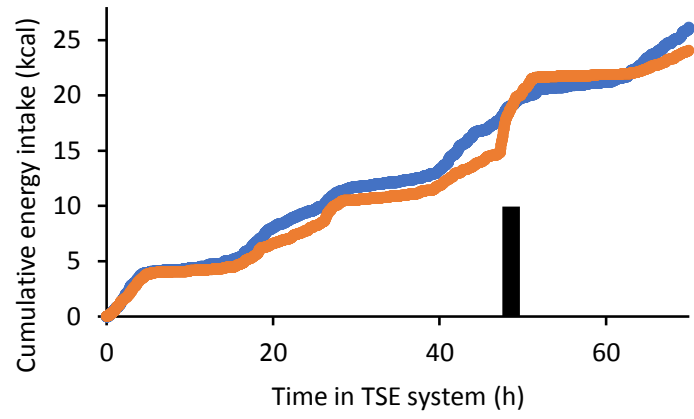

- Stock - HF diet 
FIGURE 5: MURPHY ET AL.,

AgRP

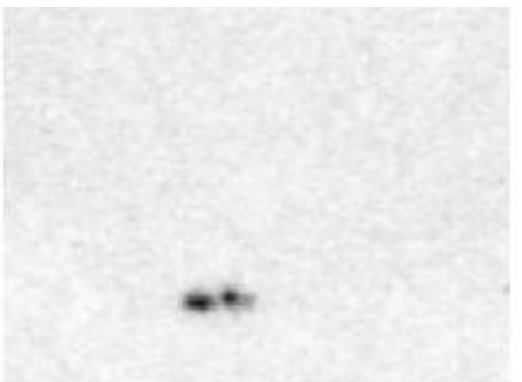

HF diet

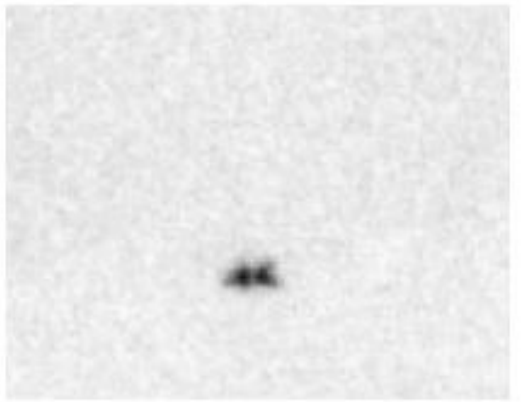

OB-Rb

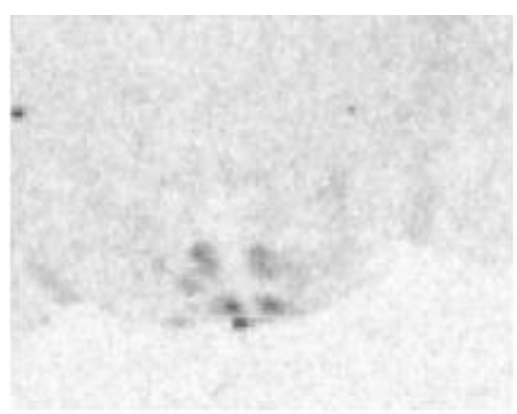

HF diet

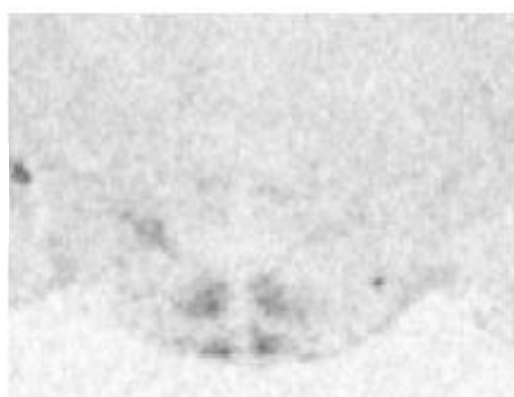

CART
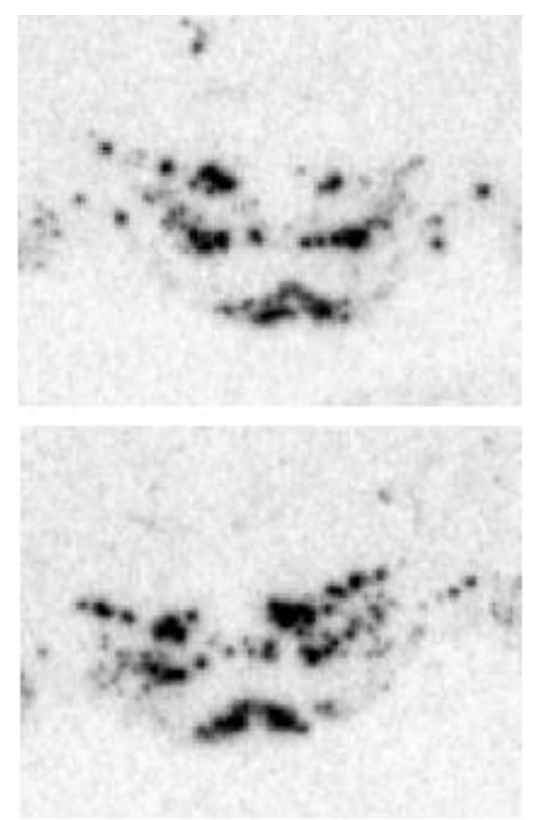

POMC
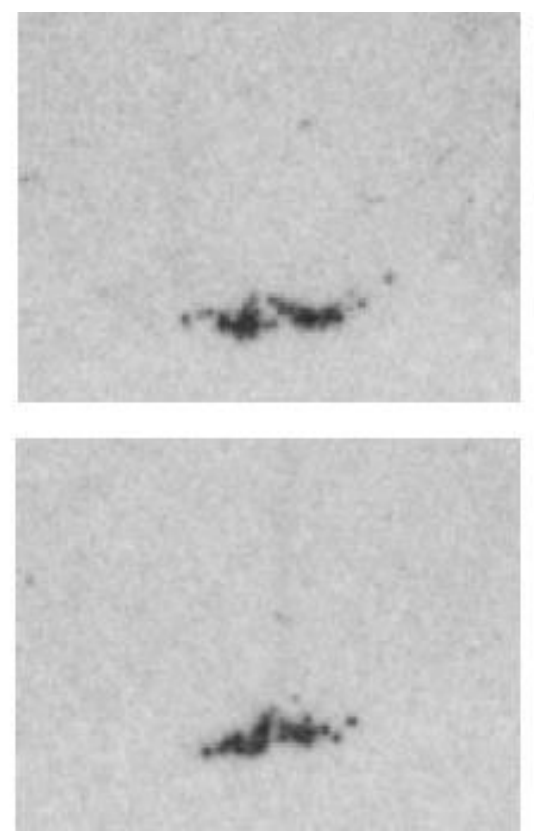

NPY
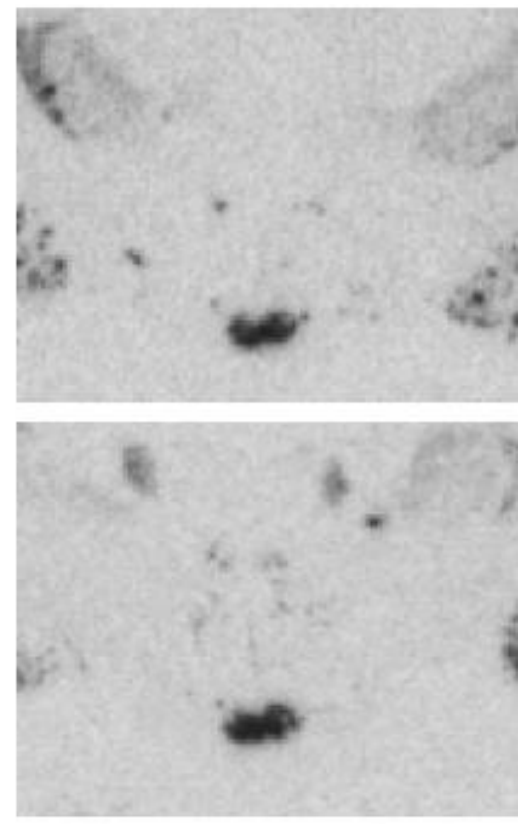

sOCS3
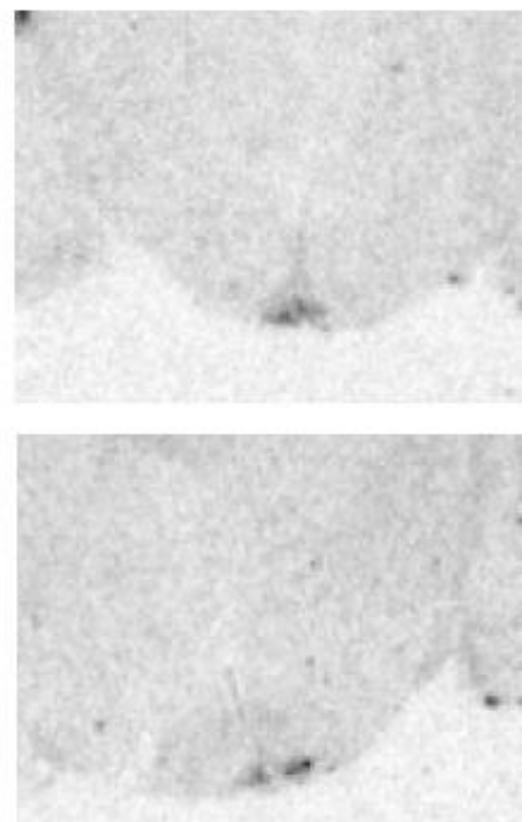
SUPP. FIGURE 1: MURPHY ET AL.,

A MALE

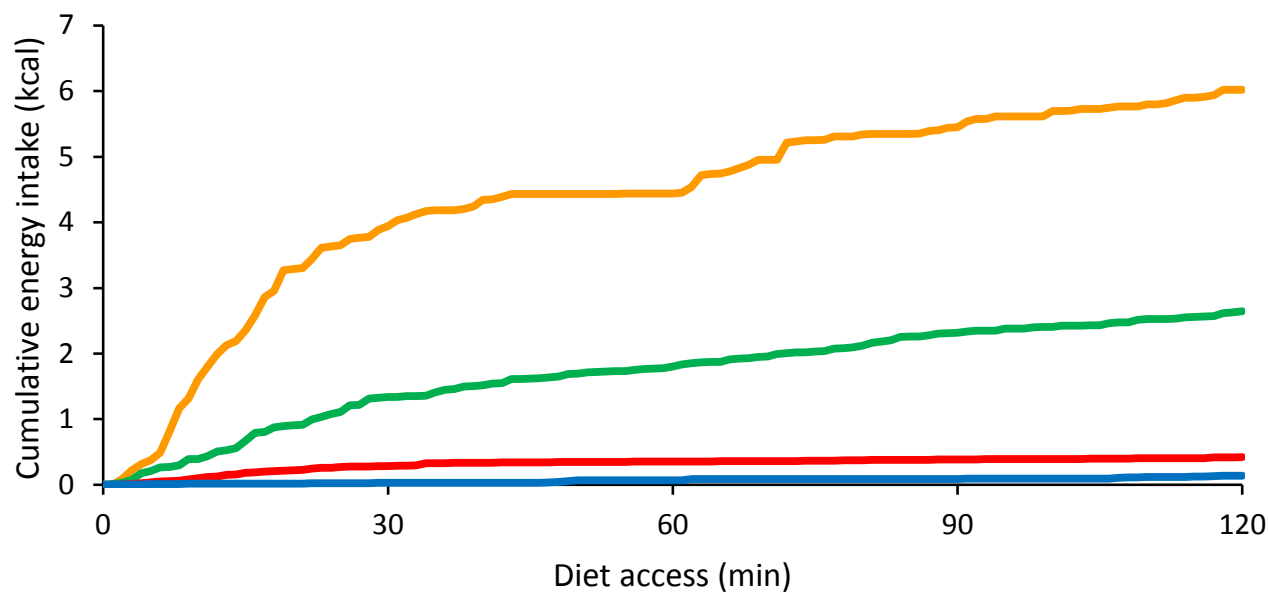

$\longrightarrow \mathrm{HF} \longrightarrow$ Ensure Sucrose $\rightleftharpoons$ Stock

\section{B FEMALE}

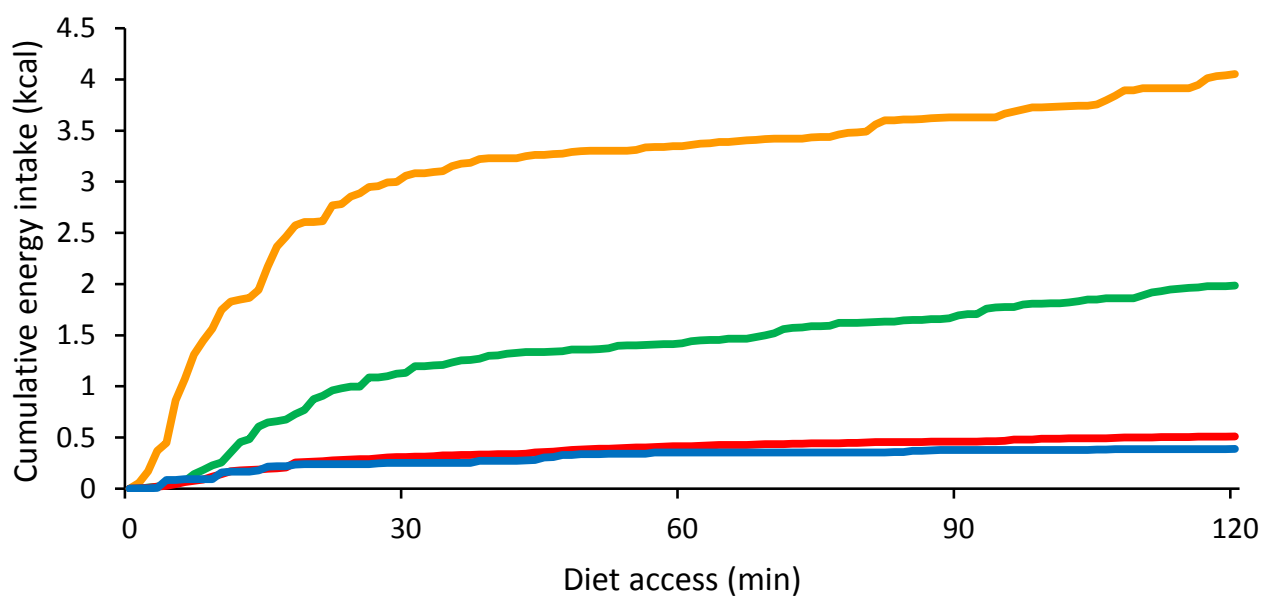

$\longrightarrow \mathrm{HF} \longrightarrow$ Ensure Sucrose $\longrightarrow$ Stock 